\title{
Successful Management of Intraoperatively Diagnosed Ruptured Spontaneous Sub-Capsular Liver Hematoma in a Woman with Preeclampsia
}

\author{
Dereje Tegene \\ Getahun Regassa \\ Aman Usu \\ Netsanet Ayalew \\ Department of Obstetrics and \\ Gynecology, Adama Hospital Medical \\ College, Adama, Ethiopia
}

Background: Ruptured subcapsular hematoma of the liver is one of the rare life-threatening complications of pregnancy and its incidence varies between 1/45,000 and 1/225,000 births. Case Presentation: A 32-year-old woman who claimed 9 months amenorrhea, presented to our hospital with elevated blood pressure and pushing down pain. After evaluation she was admitted to the labor ward with a diagnosis of latent first stage of labor and preeclampsia with severe features. She gave birth by outlet vacuum. Immediately following delivery she developed hypotension with signs of intra-abdominal fluid collection. Surgical exploration revealed massive hemoperitoneum with ruptured subcapsular hepatic hematoma. She was managed conservatively with pressure packing and drainage tube. The patient improved and was discharged after 12 days of hospital stay.

Conclusion: Timely surgical intervention, multidisciplinary team, on-time availability of blood products, and proper postoperative follow up are crucial to optimize the patient outcome.

Keywords: Adama, Ethiopia, preeclampsia, ruptured subcapsular liver hematoma

\section{Background}

Ruptured subcapsular hematoma of the liver (RSHL) in pregnancy is uncommon and occurs in $1 / 45,000$ to $1 / 225,000$ deliveries. ${ }^{1,2}$ Commonly it is associated with preeclampsia, eclampsia, or HELLP (Hemolysis Elevated Liver enzymes and Low Platelet count) syndrome. ${ }^{3}$ Women who develop a hepatic hematoma typically have epigastric pain, nausea, vomiting, and many have severe thrombocytopenia. ${ }^{4}$ If hepatic rupture occurs, swelling of the abdomen from hemoperitoneum and shock rapidly ensue. ${ }^{5}$ Management options include conservative management, packing and drainage, hepatic resection, hepatic artery ligation and liver transplantation.,

We report a successfully treated case of intraoperatively diagnosed ruptured subcapsular liver hematoma in the immediate postpartum period. It emphasizes the need for the early diagnosis, timely surgical intervention, on-time availability of blood products, and postoperative monitoring of patients. Relevant literatures were also reviewed.

\section{Case Presentation}

A 32-year-old gravid-II, Para-I woman who did not recall her last normal menstrual period (LNMP), but reported amenorrhea for the past 9 months, was referred to our 
hospital from a nearby health center with the diagnosis of latent first stage of labor (LFSOL) + preeclampsia with severity features. Her antenatal care follow up was uneventful. She complained of epigastric and right upper quadrant (RUQ) pain of 4 hours duration and pushing down pain of 2 hours. She had no abdominal trauma. Upon physical examination; her blood pressure was 170/ $100 \mathrm{mmHg}$, term-sized gravid uterus and FHB was positive, cervix $3 \mathrm{~cm}$ dilated and fully effaced. With the above finding she was given a loading dose of magnesium sulfate and admitted to the labor and delivery unit. After 30 minutes she gave birth by outlet vacuum for an indication of non-reassuring fetal heart rate pattern (fetal bradycardia), the outcome was $3.7 \mathrm{~kg}$ alive male neonate with APGAR score of 4, 4, 4, and 0 in the 1st, 5th, 10th, and 15 th minute respectively. $60 \%$ retro-placental clot was found upon post-delivery placental examination.

Immediately following delivery the woman deteri orated with the vital signs of blood pressure $=60 / 40$ $\mathrm{mmHg}$, pulse rate $=118 \mathrm{bpm}$, and abdominal distension. She was put on Pitocin drip and the uterus was well contracted with no active vaginal bleeding. Urgent transabdominal ultrasound examination showed significant fluid collection within the peritoneal cavity which was tapped under ultrasound guidance and showed frank blood. An urgent decision of surgical exploration was made and she was taken to the operating theater. The abdomen was entered through a midline incision and intraoperatively; there was significant hemoperitoneum, the uterus was intact and a large blood clot adherent to the liver surface was seen. There was also evidence of active blood oozing from the capsular breach on the right lobe of the liver surface of $8-10 \mathrm{~cm}$, suggestive of subcapsular hematoma with spontaneous rupture (Figure 1A and B).
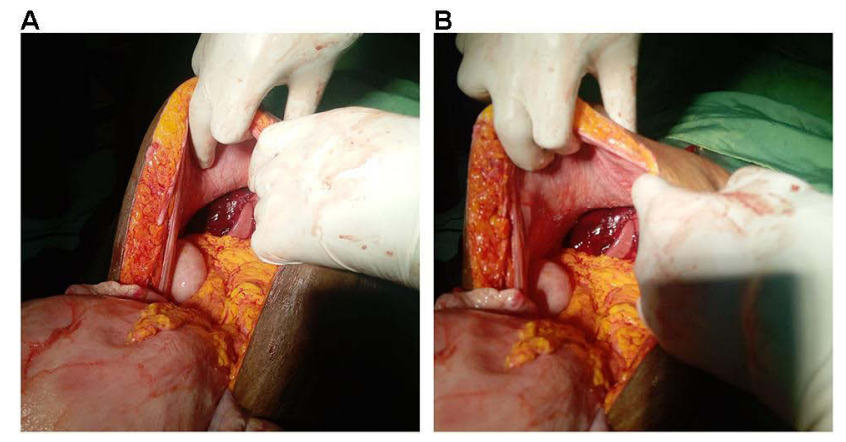

Figure I (A and B) intra-operative finding of Spontaneously Ruptured Sub-capsular Liver Hematoma.
Since we do not have a surgeon experienced in liver surgery, we consulted the general surgeon and around $2000 \mathrm{~mL}$ of hemoperitoneum was sucked out. A pack was applied over the surface to control the bleeding and a drainage tube was kept in the pelvic space. Transfusion with whole blood, platelets and fresh frozen plasma was started and the patient transferred to the recovery room. Since there was no bed in the intensive care unit, she was followed in the labor ward recovery unit. The postoperative plan was to continue transfusion, limit her ambulation, to continue magnesium sulphate, to closely follow her vital signs, and the drainage tube output.

Her laboratory studies gave the following results: serum aspartate aminotransaminase (AST), 386 IU/L; serum alanine aminotransaminase (ALT), $465.7 \mathrm{IU} / \mathrm{L}$; total bilirubin $2.3 \mathrm{mg} / \mathrm{dl}$, indirect bilirubine $1.46 \mathrm{mg} / \mathrm{dl}$; serum creatinine, $0.87 \mathrm{mg} / \mathrm{dl}$; white blood cell, 15,100 / $\mathrm{mm}^{3}$; hematocrit, $22.7 \%$; hemoglobin, $7.6 \mathrm{mg} / \mathrm{dl}$; platelet count, $409 \times 10^{3} \mu / \mathrm{mL}$. A catheterized urine specimen demonstrated proteinuria $(+++)$. From the above tests the women had partial HELLP syndrome since she had elevated liver enzymes and indirect bilirubin. On her second postoperative day, she developed abdominal distension and the drainage tube drained $700 \mathrm{~mL}$ of hemolyzed blood. She was investigated with hematocrit, $17.8 \%$; hemoglobin, $6.1 \mathrm{mg} / \mathrm{dl}$; platelet count, $209 \times 10^{3} \mu / \mathrm{mL}$. Serum electrolyte was also determined and all are within normal range.

On her 4th postoperative day the pack was removed and the patient transferred to the maternity ward. She had a smooth course in the maternity ward. On her 11th postoperative day transabdominal ultrasound examination showed a right lobe loculated collection measuring 11.2 $\mathrm{x} 11 \mathrm{~cm}$ in size extending to the subcapsular space (Figure 2). On her 12th post-operative day she was discharged home with appointment after 2 weeks. In total she was transfused with 13 units of whole blood, 10 units of platelet, and 11 units of fresh frozen plasma.

\section{Discussion}

Spontaneous hepatic bleeding is a rare condition. In the absence of trauma or anticoagulant therapy, hepatic hemorrhage may be due to underlying liver disease. The most common causes of nontraumatic hepatic hemorrhage are hepatocellular carcinoma (HCC) and hepatocellular adenoma. Other liver tumors, such as focal nodular hyperplasia (FNH), hemangioma, and metastases can also cause hepatic hemorrhage. HELLP syndrome, amyloidosis, and 


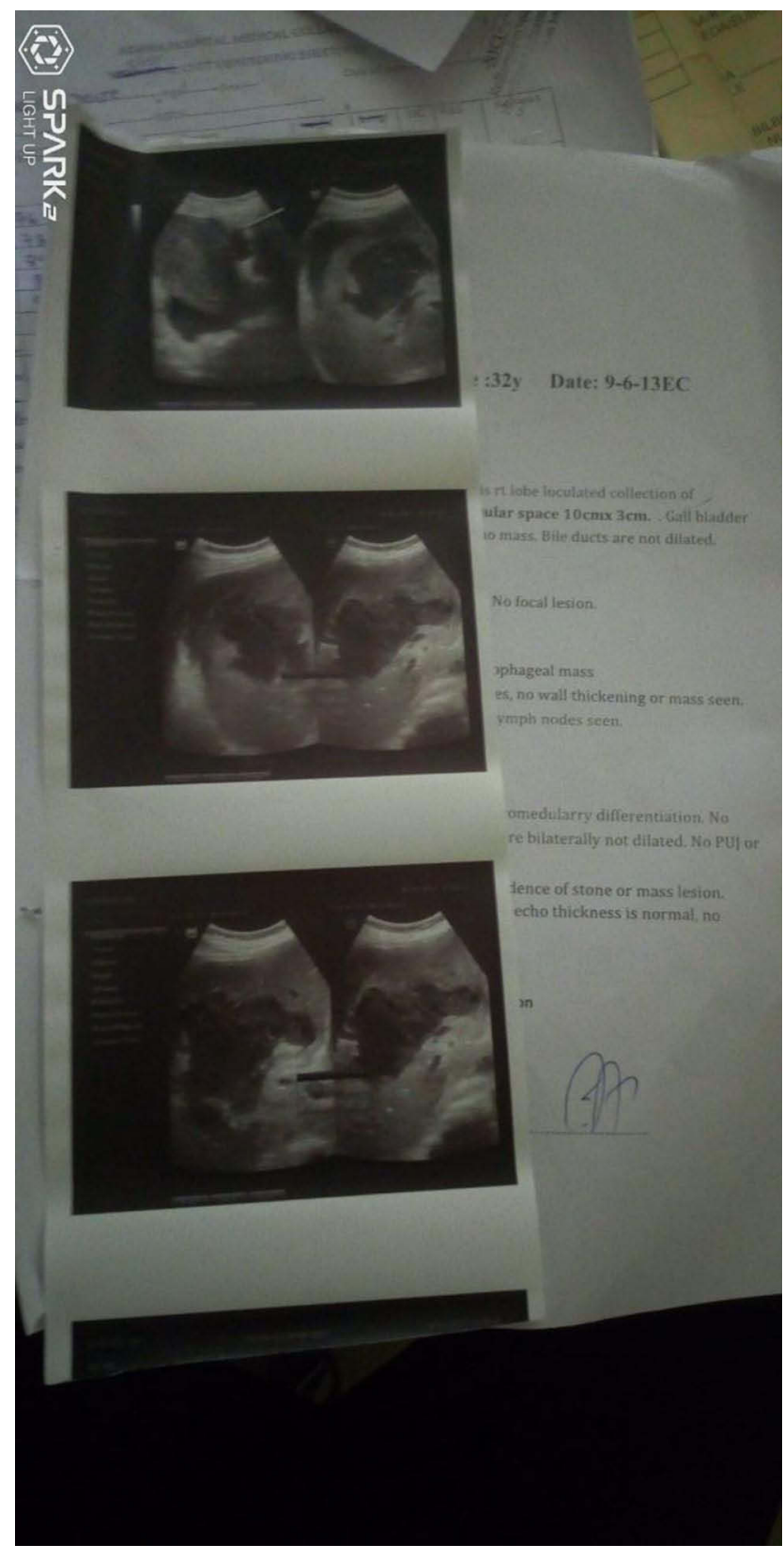

Figure 2 Abdominal Ultrasound showing Ruptured Sub-capsular Liver Hematoma.

miscellaneous causes are other conditions associated with spontaneous hepatic hemorrhage. ${ }^{7}$

The liver is not primarily involved in preeclampsia, and hepatic involvement is observed in only $10 \%$ of women with severe preeclampsia. ${ }^{8}$ One of the rare and potentially fatal complications of HELLP syndrome is subcapsular liver hematoma (SLH), which occurs in about $1-2 \%$ of all patients with preeclampsia and HELLP syndrome. ${ }^{5,9}$ In women with HELLP syndrome, the pathogenesis of SLH is not well understood. Fibrin deposition leading to platelet activation, thrombus formation, and occlusion of capillaries, which ultimately progresses to hepatic necrosis and hemorrhage may be involved. ${ }^{5,10}$ Hepatic rupture is the most concerning and potentially fatal complication of SLH, and its incidence varies from $1 / 45,000$ to $1 / 225,000$ deliveries. ${ }^{1,2}$

Ruptured SLH is commonly associated with preeclampsia, eclampsia, or HELLP. Other factors that may increase the risk of ruptured SLH include traumatic events in the setting abdominal palpation, manual removal of the placenta, and uterine contractions. ${ }^{3}$ Hepatic rupture due to SLH most commonly involves the right lobe of the liver. Maternal mortality of SLH ranges from $17 \%$ to $59 \%$, depending on rupture of the hematoma, timing of diagnosis, and availability of therapeutic interventions. ${ }^{5}$ A perinatal mortality rate is also reported in literature as $42 \% .^{10}$

Patients with SCLH commonly present with severe persistent epigastric or right upper quadrant (RUQ) pain, nausea, vomiting and features of severe thrombocytopenia. ${ }^{8}$ The presenting symptoms and signs of ruptured SLH are subtle and non-specific. Most of the patients commonly present with abdominal pain, shoulder pain, hypovolemic shock, evidence of intra-abdominal collection, respiratory difficulty, and often with a dead fetus. $^{5,11}$ The development of profound hypovolemic shock in a previously hypertensive patient is the hallmark of hematoma rupture. ${ }^{5}$ In general a high index of clinical suspicion is necessary to make the diagnosis, accordingly emergency abdominal ultrasonography, CT, or magnetic resonance imaging should be considered. Paracentesis can confirm intraperitoneal bleeding. ${ }^{3}$ The diagnosis may also be made by direct visualization of free blood in the abdominal cavity and identification of the liver lesion at the time of caesarean delivery for severe PE. In a patient presenting with hemodynamic instability and acute abdominal pain, the diagnosis is usually made during an emergency laparotomy. ${ }^{12}$

The presence of ruptured subcapsular liver hematoma that results in shock is a surgical emergency that requires acute multidisciplinary treatment. ${ }^{6}$ Resuscitation should consist of massive transfusions of blood, correction of coagulopathy with FFP and platelets, and immediate laparotomy. A team experienced in liver trauma surgery should be consulted. If hepatic rupture is suspected, an incision in the upper abdomen is necessary for adequate surgical exposure. Options at laparotomy include packing and drainage, surgical ligation of the hemorrhaging hepatic segments, embolization of the hepatic artery to the involved 
liver segment, loose suturing of the omentum or surgical mesh to the liver to improve integrity, and partial liver resection. ${ }^{5,6}$ Liver transplantation as a last resort must be considered, in patients with hepatic failure or uncontrollable hepatic hemorrhage. ${ }^{6}$

In this case, the patient had no history of trauma or liver disease. She was diagnosed with preeclampsia with severity features because of her severe hypertension and persistent severe epigastric and right upper quadrant pain just intrapartum. Even though the platelet count was within normal range, the liver enzyme and indirect bilirubin were elevated; this goes with the diagnosis of partial HEELP syndrome. Most patients with ruptured subcapsular liver hematoma had significantly elevated liver enzymes (ALT \& AST), which was also seen in our case.

In addition to preeclampsia and HEELP syndrome, our case had strong frequent uterine contractions resulting in precipitated labor which can also contribute for the development of this complication. Imaging plays a significant role in the diagnosis and management of this potentially lethal entity. Computed tomography (CT), or magnetic resonance (MR) imaging have been suggested by many experts for diagnosis, management and follow up of patients with hemorrhagic liver lesion. ${ }^{7}$ In our case these imaging modalities were not available at our hospital and the patient could not afford the cost at private facility.

The diagnosis of ruptured sub-capsular liver hematoma was made intraoperatively in our case. The diagnosis of ruptured sub-capsular liver hematoma is usually made during an emergency laparotomy, in a patient presenting with hemodynamic instability and acute abdominal pain. ${ }^{12}$ A large hematoma was on the right lobe of the liver in this patient. Perihepatic packing was carried out to control the bleeding and a drainage tube was kept in the pelvic space. Loose suturing of the omentum or surgical mesh to the liver to improve integrity, surgical ligation of the hemorrhaging hepatic segments, and partial liver resection are the clinical options. ${ }^{5,6}$

\section{Conclusions}

In conclusion, ruptured sub-capsular hematoma of the liver is a rare but potentially life-threatening complication of pregnancy. High index of clinical suspicion is very important for early recognition and treatment which significantly improves the patient outcomes. Timely surgical intervention, multidisciplinary team approach, on-time availability of blood products, and proper postoperative follow up are crucial to improve the patient outcome.

\section{Abbreviations}

AHMC, Adama Hospital Medical College; ALT, alanine aminotransaminase; AST, aspartate aminotransaminase; CT, computer tomography; HELLP, hemolysis elevated liver enzymes and low platelet syndrome; ICU, intensive care unit; SLH, sub-capsular liver hematoma.

\section{Data Sharing Statement}

Data used in this case report can be obtained from the corresponding author.

\section{Ethics Approval}

We obtained ethical clearance from the Institutional Ethics Review Board of Adama Hospital Medical College.

\section{Consent for Publication}

Written informed consent was provided by the patient for the publication of this case report and accompanying images.

\section{Author Contributions}

The conception of the report was developed by the corresponding author. All authors took part in drafting, execution, acquisition, and interpretation of data. On the preparation of the document, all authors were involved in the literature review and critically reviewing the article. All authors read and gave final approval of the submitted document and have agreed to be published on the submitted journal. All authors agreed to take responsibility and be accountable for the contents of the article.

\section{Funding}

There is no funding to report.

\section{Disclosure}

The authors declare that they have no competing interests.

\section{References}

1. Rinehart BK, Terrone DA, Magann EF, et al. Preeclampsia-associated hepatic hemorrhage and rupture: mode of management related to maternal and perinatal outcome. Obstet Gynecol Surv. 1999;54 (3):196-202. doi:10.1097/00006254-199903000-00024

2. Wicke C, Pereira PL, Neeser E, et al. Subcapsular liver hematoma in HELLP syndrome: evaluation of diagnostic and therapeutic options-a unicenter study. Am J Obstet Gynecol. 2004;190(1):106-112. doi:10.1016/j.ajog.2003.08.029

3. Zhou X, Zhang M, Liu Z, et al. A rare case of spontaneous hepatic rupture in a pregnant woman. BMC Pregnancy Childbirth. 2018;18 (1):1-4. doi:10.1186/s12884-018-1713-5

4. Gabbe SG, Niebyl JR, Simpson JL, et al. Obstetrics: Normal and Problem Pregnancies e-Book. Elsevier Health Sciences; 2016. 
5. Ditisheim A, Sibai BM. Diagnosis and management of HELLP syndrome complicated by liver hematoma. Clin Obstet Gynecol. 2017;60(1):190-197. doi:10.1097/GRF.0000000000000253

6. Wilson $\mathrm{S}$, White $\mathrm{AD}$, Young $\mathrm{AL}$, et al. The management of the surgical complications of HELLP syndrome. Ann R Coll Surg Engl. 2014;96(7):512-516. doi:10.1308/003588414X13946184901362

7. Casillas VJ, Amendola MA, Gascue A, et al. Imaging of nontraumatic hemorrhagic hepatic lesions. Radiographics. 2000;20 (2):367-378. doi:10.1148/radiographics.20.2.g00mc10367

8. Landon MB, Galan HL, Jauniaux ERM, et al. Obstetrics: Normal and Problem Pregnancies E-Book. Elsevier Health Sciences; 2020.

9. Karateke A, Silfeler D, Karateke F, et al. HELLP syndrome complicated by subcapsular hematoma of liver: a case report and review of the literature. Case Rep Obstet Gynecol. 2014;2014:1-3. doi:10.1155/ $2014 / 585672$
10. Marsh FA, Kaufmann S, Bhabra K. Surviving hepatic rupture in pregnancy-a literature review with an illustrative case report. J Obstet Gynaecol. 2003;23(2):109-113. doi:10.1080/0144361 031000074583

11. Ghorbanpour M, Makarchian HR, Yousefi B, et al. Conservative Management of Postpartum HELLP syndrome and Intraparenchymal liver hematoma; a case report. Bull Emerg Trauma. 2019;7(2):196. doi:10.29252/beat-070218

12. Ngene N, Amin N, Moodley J. Ruptured subcapsular hematoma of the liver due to pre-eclampsia presenting as interstitial pregnancy and the role of intra-abdominal packing. Niger J Clin Pract. 2015;18 (2):300-303. doi:10.4103/1119-3077.151074

\section{Publish your work in this journal}

The International Journal of Women's Health is an international, peerreviewed open-access journal publishing original research, reports, editorials, reviews and commentaries on all aspects of women's healthcare including gynecology, obstetrics, and breast cancer. The manuscript management system is completely online and includes a very quick and fair peer-review system, which is all easy to use. Visit http://www.dovepress.com/testimonials.php to read real quotes from published authors. 